\title{
Results from ND280 near detector
}

\author{
Joanna Zalipska, ${ }^{1, a}$, on behalf of the T2K collaboration \\ ${ }^{1}$ National Centre for Nuclear Research, Ho'za 69, 00-681 Warsaw, Poland
}

\begin{abstract}
Since the beginning of operation of the Tokai to Kamioka experiment (T2K) the Near Detector system (ND280) has been collecting neutrino interactions. It was able to collect large statistics of data within a few years of operation of the T2K experiment because of high beam intensity and its close location to the production point, a distance of the $280 \mathrm{~m}$ from the production target. This paper presents results of a measurement of charged current interactions in ND280 which are used to suppress systematic errors in the oscillation analysis. Systematic effects due to uncertainty in the beam profile and cross sections of neutrino interactions are also discussed.
\end{abstract}

\section{The T2K experiment}

The T2K experiment is a long-baseline neutrino oscillation experiment which can provide precise measurements of atmospheric neutrino oscillation parameters such as $\Delta m_{23}^{2}$ and $\theta_{23}$ [1] by studying the disappearance of $v_{\mu}$ from the beam. It was also the first experiment to observe the appearance of $v_{e}$ related to the mixing angle $\theta_{13}$ [2,3]. TT2K [4] is located in Japan and uses a neutrino beam produced by the J-PARC accelerator complex in Tokai. The $v_{\mu}$ beam is produced in a conventional way from $30 \mathrm{GeV}$ protons provided by the accelerator when they hit a graphite target. The beam is sent to a far detector which is located $295 \mathrm{~km}$ away from the production point. A characteristic of this experiment is that the far detector sits $2.5^{\circ}$ off the beam axis. Such a configuration assures a narrow energy spectrum of the beam centered at $600 \mathrm{MeV}$ at the oscillation maximum. It also suppresses the high-energy tail of the neutrino beam which reduces background coming from intrinsic electron neutrinos. Since a good knowledge of neutrino flux is crucial for precise measurement of oscillation parameters, the T2K is equipped with two near detectors: the ND280 and INGRID. They provide measurements of the produced neutrino interaction rate and monitor the position of the beam center. The T2K uses Super-Kamiokande (SK) [5] as the far detector aimed to study neutrino oscillation signal. The SK relies on the water Cherenkov technique to detect charged particles coming out of a neutrino interaction. Cherenkov light emitted by those particles is detected by 11000 photo-multiplier tubes. By studying the ring images created by the emitted light, we are able to identify the type of interacting particle and consequently say what was the flavor of interacting neutrino: $v_{e}$ or $v_{\mu}$.

\footnotetext{
ae-mail: joanna.zalipska@fuw.edu.pl
} 


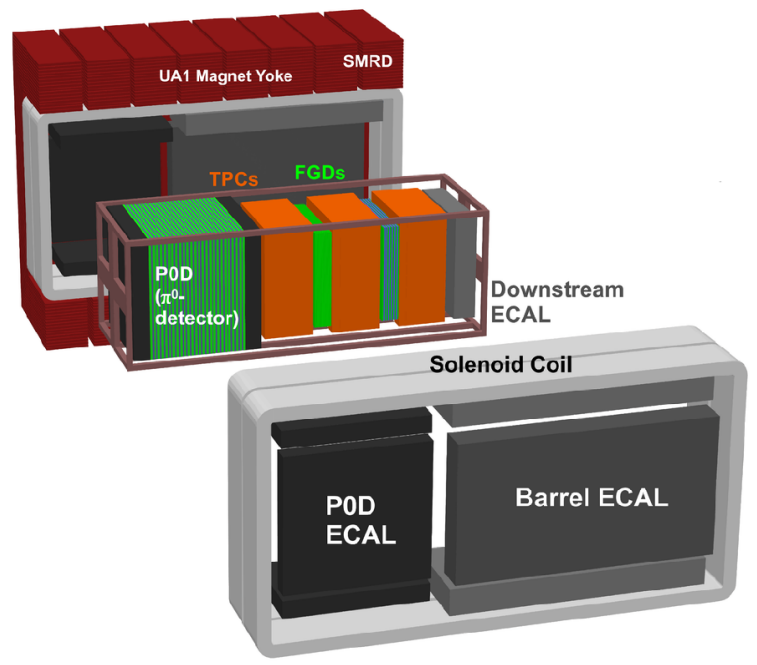

Figure 1. Schematic of the ND280 detector system.

\section{The ND280}

\subsection{Detector}

The ND280 is dedicated to study the un-oscillated spectrum of neutrinos $280 \mathrm{~m}$ from the production target. Same as the far detector, it is located at $2.5^{\circ}$ off-axis since it provides a reference measurement of the neutrino flux used by the far detector for oscillation analysis. The detector is placed inside a magnet which provides a $0.2 \mathrm{~T}$ magnetic field as shown in Fig. 1.

The main component of the ND280 is the tracker detector with gas TPC [6] modules interlaid with scintillator sub-detector modules called Fine-Grained Detectors (FGD) [7]. The first module of FGD1 acts as a target for neutrino interactions on carbon. The second FGD2 module is equipped with water layers therefore it may also provide a measurement of neutrino interaction on water, since it has the same target as that used by the far detector. Thanks to its thin scintillation bars of $1 \mathrm{~cm}$ width the FGD can provide precise vertex information of the interacting neutrino. The gaseous TPCs are used to measure momenta of charged particles by studying track curvature in the magnetic field reaching inverse momentum resolution of $0.1(\mathrm{GeV} / \mathrm{c})^{-1}$. They can also provide particle identification by measuring energy loss and distinguish muons from electrons, charged pions and protons.

A separate detector P0D (Pizero Detector) was build to study neutral current (NC) interactions with $\pi^{0}$ coming out of the vertex. It is placed upstream of the tracker and it is composed of scintillator modules interlaid with lead and water layers.

The inner part of the detector is surrounded by an electromagnetic calorimeter (ECal) which can tag escaping electrons or positrons from $\pi^{0}$ decay.

Additionally, the scintillator slabs of the side muon range detector (SMRD) are placed inside the magnet yoke. They are used to tag cosmic ray muons which enter the detector or detect muons which escape the inner volume. 


\subsection{Goals}

The main goal of the ND280 measurement is to reduce the systematic uncertainties associated with flux prediction and neutrino interaction cross sections for prediction of number of events expected in the far detector, Super-Kamiokande, for the $v_{\mu}$ and $v_{e}$ oscillation analysis. Therefore the effort of ND280 analysis is concentrated on measurements of the un-oscillated $v_{\mu}$ flux, the $v_{e}$ beam contamination (being one of the sources of background for the search of $v_{e}$ appearance in the $v_{\mu}$ beam) and the neutrino interaction cross sections on oxygen.

The approach to the oscillation analysis relies on the prediction of event rates in the SK in order to compare them with measured values. To predict those rates our knowledge about neutrino flux models and cross sections is used. For that purpose the proton interactions with the target are modeled using FLUKA inside the target and GEANT3 elsewhere. The production of $\pi$ and $K$ induced by the proton beam has been verified by the NA61/SHINE experiment which performed at CERN a measurement of proton beam interactions with carbon thin $[8,9]$. As a result the total uncertainty of the neutrino flux at the order of $10 \%$ was reached. Then the neutrino interactions are simulated using NEUT Monte Carlo generator [10] for the near and far detector. The NEUT cross section models were tuned to external data from lepton and pion scattering experiments as well to the neutrino experiment MiniBooNE allowing to verify such parameters as axial mass, Fermi momentum and binding energy. Finally the ND280 measurement is used to validate all those models which were used to predict the neutrino flux.

\subsection{Analysis overview}

The ND280 analysis considers interactions of $v_{\mu}$ by searching for their signature through charged current processes (CC). To be selected as a candidate for $\mathrm{CC}$ interaction an event is required to have a vertex located in the FGD1. It must also have a negatively charged track crossing the TPC2 sub-detector where particle identification provided by the TPC is consistent with a muon hypothesis. Furthermore the $\mathrm{CC}$ sample is divided into three categories depending on the number of pions leaving the nucleus after the interaction. The following topologies of an event are considered: CC-O-pion, CC-1-pion and CC-other. The CC-O-pion sample is dominated by charged current quasi-elastic interactions (CCQE) when there are no pions in the final state. Events with one positive pion track are classified into the CC-1-pion sub-sample. In the case of long tracks crossing TPC, a particle identification is used to identify pions. However, also particles which do not leave the FGD are considered. If they travel a long enough distance in FGD to be reconstructed as a track, their type is found using FGD particle identification procedure which can distinguish pions from short proton tracks. Those decaying pions are also tagged by detecting the delayed signal associated with a Michel electron. The third defined class of events, $C C$-other, contains the remaining interactions which have more than one positive pion or have at least one negative or neutral pion exciting the interaction.

To summarize, the CC-0-pion sub-sample has a $47.8 \%$ efficiency and a $72.4 \%$ purity and indeed is enhanced with CCQE processes which remain in $63.3 \%$ of interactions in that event category. In contrast the CC-1-pion sample is dominated by resonant interactions (RES) 39.4\%. It has an efficiency of $28.4 \%$ and purity of $51.4 \%$. The last sub-sample CC-other has an efficiency and a purity equal $29.7 \%$ and $73.6 \%$ respectively. Here the dominant interaction type is deep inelastic processes (DIS) which account for as much as $67.7 \%$ of the $C C$-other sub-sample.

For each defined sub-sample in data and Monte Carlo, the two-dimensional plots of muon momentum and angle are prepared. Then the fit is performed which varies parameters related to neutrino spectrum and cross sections to obtain the best agreement between data and simulation. The accumulated protons on target used for that analysis is $5.90 \cdot 10^{20}$ and corresponds to the data taken until 2013. 

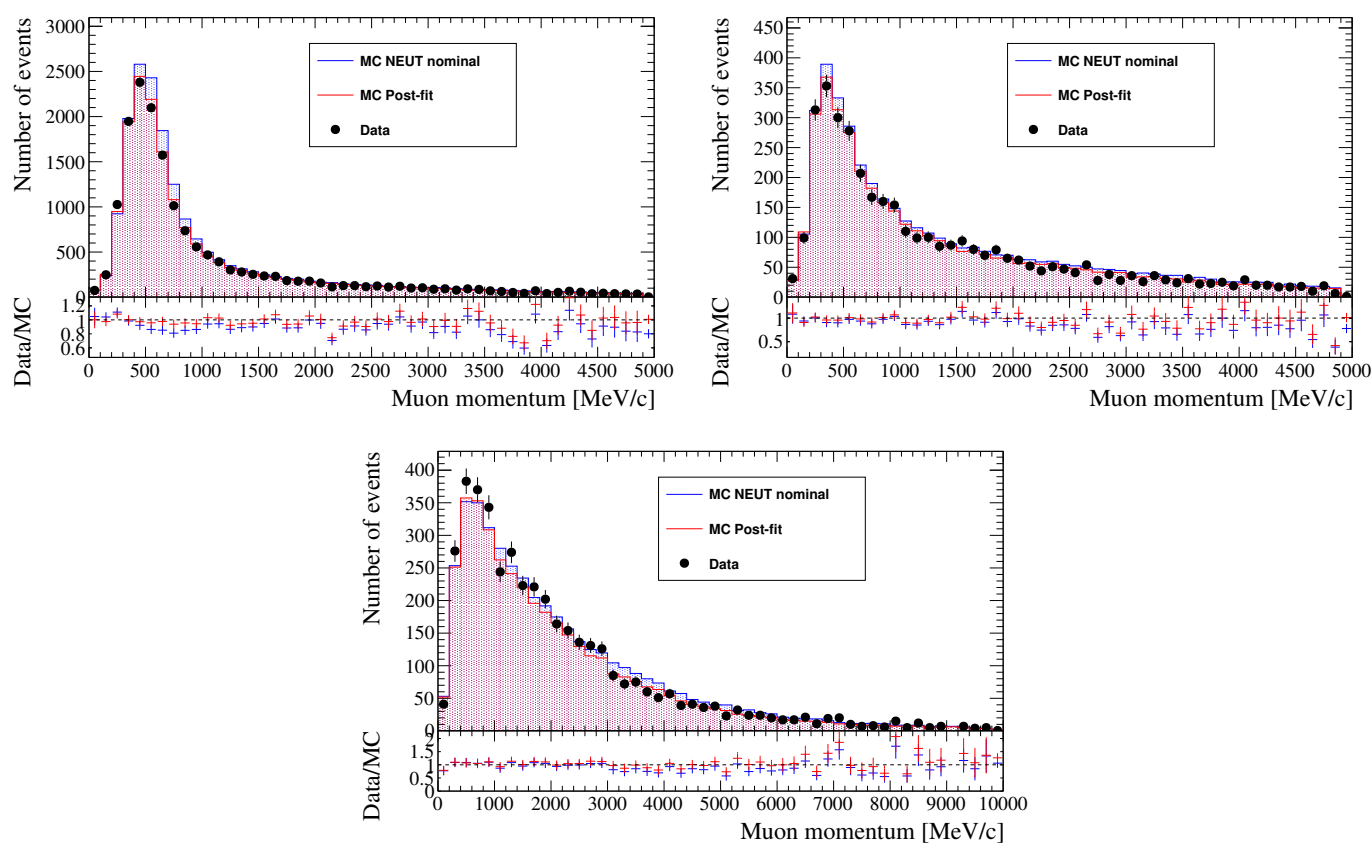

Figure 2. Distribution of reconstructed muon momenta for the selected samples of CC-0-pion (upper left), $C C$ 1-pion (upper right) and $\mathrm{CC}$-other (lower) samples. Blue histograms show the MC distribution before the fit, while the red one shows the results after the fit.

\section{Near Detector measurements}

\subsection{Constraining flux and cross sections}

The agreement between ND280 data and Monte Carlo after performing the fit is really good as demonstrated on by the distribution of reconstructed muon momentum in Fig. 2. As a consequence the ND280 measurement can more precisely predict the expected neutrino spectra at the SuperKamiokande far detector. In Fig. 3 the uncertainty on the flux for each bin of the neutrino energy spectrum at SK is plotted separately for $v_{\mu}$ and $v_{e}$. It is clear that performed measurement reduces the uncertainty for each energy bin.

As was described in section 2.2 the NEUT generator of neutrino interactions includes parameters such as axial mass whose nominal values were set to $M_{A}^{Q E}=1.21 \pm 0.45 \mathrm{GeV}$ and $M_{A}^{R E S}=1.41 \pm$ 0.22 . Thanks to treating separately samples enhanced with CCQE interactions and those with pion production, we were able not only to fit the central value of those parameters but also significantly reduce their errors to following values $M_{A}^{Q E}=1.223 \pm 0.072 \mathrm{GeV}$ and $M_{A}^{R E S}=0.963 \pm 0.063$. Also normalization factors for CCQE, $\mathrm{CC} 1 \pi$ and $\mathrm{NC} 1 \pi^{0}$ interactions were obtained as $0.961 \pm 0.076,1.22 \pm$ 0.16 and $1.10 \pm 0.25$ respectively.

\subsection{Electron neutrino beam contamination}

According to the beam simulation contamination from electron neutrinos from the beam make up $1.2 \%$ of the beam contamination at the peak energy of $600 \mathrm{MeV}$. The following measurement by 

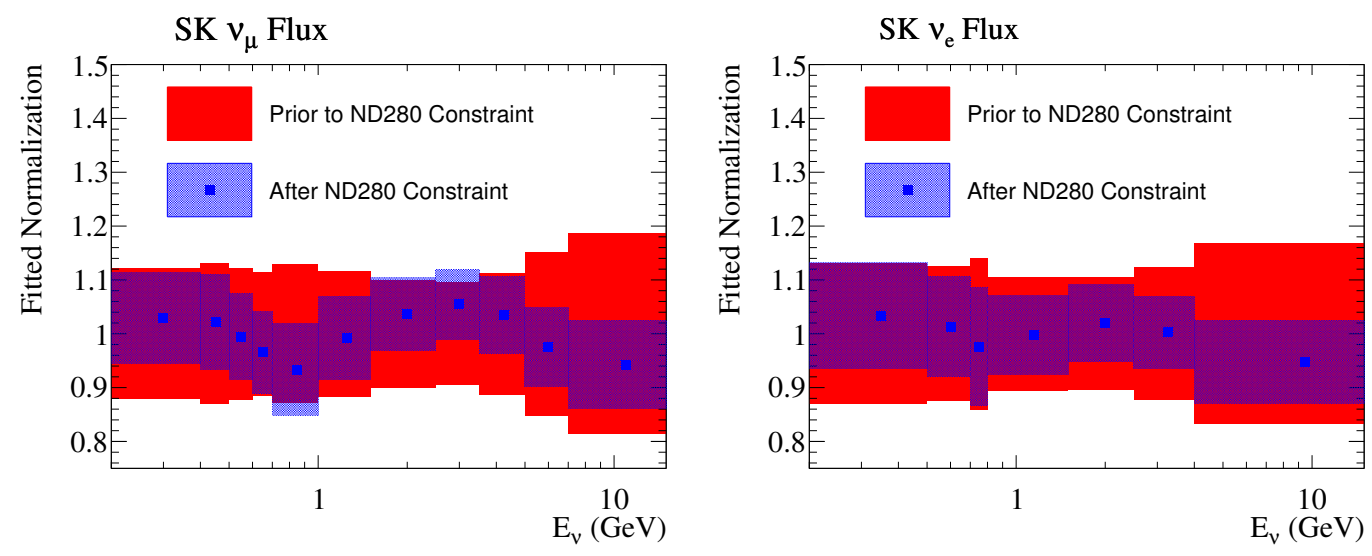

Figure 3. Precision of flux prediction for muon neutrinos (left) and electron neutrinos (right) at SuperKamiokande. Red indicates the error before the fit and blue after the fit.

the ND280 was performed to verify this prediction. The selection of the events originating from $v_{e}$ is the same as for the measurement of CC interactions of $v_{\mu}$ except that a negative electron track is required to be found in TPC, being a signature of $\mathrm{CC} v_{e}$ interaction. Then the sample is split into two categories: the CC-0-pion and CC-1-pion +CC-other. The first one is defined by events with only one reconstructed electron track and therefore it is predominantly CCQE interactions. All other events containing resonances and DIS processes end up in the second sub-sample. The main background for $v_{e}$ interactions is associated with $\gamma$. When a $\gamma$ converts into an electron and a positron it will be identified in TPC as an event containing electron track. A likelihood fit between data and Monte Carlo is performed for those sub-samples. As a result it provides a ratio of $v_{e}$ beam contamination in CC-0-pion sample of:

$$
R\left(v_{e}, \mathrm{CC}-0-\text { pion }\right)=1.10 \pm 0.14(\text { stat } .) \pm 0.10(\text { syst } .)
$$

and in the other samples of CC-1-pion $+C C$-other of:

$$
R\left(v_{e}, \mathrm{CC}-1-\text { pion }+\mathrm{CC}-\text { other }\right)=1.03 \pm 0.11(\text { stat } .) \pm 0.12(\text { syst } .)
$$

These obtained results are consistent with the predicted $v_{e}$ beam contamination. Details of this analysis were published in [11].

\section{Effect of ND280 measurement on the far detector analysis}

Knowledge derived from the ND280 measurement is directly used for the analysis of $v_{e} \rightarrow v_{\mu}$ oscillation in the far detector Super-Kamiokande. It affects the estimate of the expected number of electron events as summarized in Table 1. We obtained that number from predicted number of events distribution made under the assumption of oscillation parameters as given in Table 1. Such a distribution is significantly more narrow when the ND280 constraint is used, therefore our prediction of electron events becomes more precise. Table 1 shows how the systematic uncertainty related to flux and cross sections on the number of expected electron events is affected by the measurement performed by the near detector. When no ND280 information was used the uncertainty was $26.5 \%$ 
Table 1. Predicted number of events at the far detector induced by interaction of electron neutrinos and its uncertainty associated with flux and cross sections for two values of oscillation parameter $\sin ^{2} 2 \theta_{13}=0.0$ and $\sin ^{2} 2 \theta_{13}=0.1$.

\begin{tabular}{|l|c|c|c|c|}
\hline & \multicolumn{2}{|c|}{$\sin ^{2} 2 \theta_{13}=0.0$} & \multicolumn{2}{c|}{$\sin ^{2} 2 \theta_{13}=0.1$} \\
& $\begin{array}{c}v_{e} \text { prediction } \\
\text { (events) }\end{array}$ & $\begin{array}{c}\text { Error from } \\
\text { constraint } \\
\text { parameters }\end{array}$ & $\begin{array}{c}v_{e} \text { prediction } \\
\text { (events) }\end{array}$ & $\begin{array}{c}\text { Error from } \\
\text { constraint } \\
\text { parameters }\end{array}$ \\
\hline No ND280 constraint & 5.3 & $22.0 \%$ & 22.6 & $26.5 \%$ \\
ND280 constrained 2012 & 5.1 & $6.1 \%$ & 21.6 & $4.7 \%$ \\
ND280 constrained & 4.6 & $4.9 \%$ & 20.4 & $3.0 \%$ \\
\hline
\end{tabular}

Table 2. Systematic uncertainty on number of predicted $v_{e}$ events at SK for two values of oscillation parameter $\sin ^{2} 2 \theta_{13}=0.0$ and $\sin ^{2} 2 \theta_{13}=0.1$.

\begin{tabular}{|l|c|c|}
\hline Error source & $\sin ^{2} 2 \theta_{13}=0.0$ & $\sin ^{2} 2 \theta_{13}=0.1$ \\
\hline Flux + cross sections & $4.9 \%$ & $3.0 \%$ \\
Other cross sections & $6.7 \%$ & $7.5 \%$ \\
SK + FSI & $7.3 \%$ & $3.5 \%$ \\
\hline Total & $11.1 \%$ & $8.8 \%$ \\
Total (2012) & $13.0 \%$ & $9.9 \%$ \\
\hline
\end{tabular}

$(22.0 \%)$ for the assumed mixing angle of $\sin ^{2} 2 \theta_{13}=0.1\left(\sin ^{2} 2 \theta_{13}=0.0\right)$. With a recent analysis of ND280 data that error can be reduced to as low as 3.0\% (4.9\%). Furthermore, it is demonstrated that the effect on the ND280 analysis associated with new reconstruction and selection which takes into account pions produced in the interaction, introduced in analysis in 2013 allowed a reduction in the uncertainty from $4.7 \%(6.1 \%)$. When the total error on $v_{e}$ analysis is considered, as summarized in Table 2, contribution associated with flux and cross sections measurements in the near detector is the smallest. The most dominant error is coming from measurements in the SK detector and uncertainties associated with cross section parameters which are not probed by recent measurements of ND280.

Similarly Table 3 was made for the disappearance analysis of muon neutrinos assuming the best fit value of the oscillation parameters of $\left(\sin ^{2} \theta_{23}, \Delta m_{23}^{2}\right)=\left(0.5,2.4 \cdot 10^{-3}\right)$. The conclusion is the same as for $v_{e}$ analysis that the ND280 error is lowest and two other errors are dominant. Here when the near detector constraint would not be used the size of the error from the flux and cross sections would be as large as $21.8 \%$ instead of current value of $2.7 \%$.

There is still some room for improvement associated with the measurements done by the near detector system. The ND280 should be able to reduce the size of the error coming from the other cross sections since it will provide measurements of the neutrino cross section on water target when analysis of the FGD2 data is finalized along with measurements provided by the P0D.

\section{Other measurements in ND280}

This paper covers measurements of neutrino flux and cross section parameters used for the oscillation analysis. However the ND280 data are also used to perform flux averaged differential cross section measurements. The results of measurements of $v_{\mu} \mathrm{CC}$ and $v_{e} \mathrm{CC}$ inclusive cross sections were already published in [12] and [13] respectively. As was mentioned in the previous section, there is ongoing work on the measurement of neutrino interactions on the water targets in the second 
Table 3. Systematic uncertainty on number of predicted $v_{\mu}$ events at SK for the best value of fitted oscillation parameters.

\begin{tabular}{|l|c|}
\hline Error source & $\left(\sin ^{2} \theta_{23}, \Delta m_{23}^{2}\right)=\left(0.5,2.4 \cdot 10^{-3}\right)$ \\
\hline Flux + cross sections & $2.7 \%$ \\
Other cross sections & $4.9 \%$ \\
SK + FSI & $5.6 \%$ \\
\hline Total & $8.1 \%$ \\
\hline
\end{tabular}

module of the FGD. Among other analysis, we are trying to provide measurements of charged pion production and multi nucleon interactions. The last ones are neutrino interactions which occur on two nucleons simultaneously. They have not yet been considered for published analyzes performed by T2K. However they are studied with NuWro [14] generator and they were recently added into NEUT Monte Carlo generator as well.

In June $2014 \mathrm{~T} 2 \mathrm{~K}$ started to take data in anti-neutrino mode to study CP violation by measuring oscillations in $\bar{v}_{e}$ appearance and $\bar{v}_{\mu}$ disappearance. Since the far detector is not magnetized it can not discriminate between $\mu^{+}$and $\mu^{-}$and consequently is not able to distinguish between interactions of $v_{\mu}$ and $\bar{v}_{\mu}$. Therefore, precise knowledge about anti-neutrino cross section is crucial. The data collected by the ND280 will be used to measure cross section of $\bar{v}_{\mu}$ interactions.

\section{Conclusions}

The ND280 demonstrated its importance for the oscillation analysis of $v_{e}$ and $v_{\mu}$ performed by the T2K experiment. Thanks to thousands of accumulated $v_{\mu}$ interactions it constrains the neutrino flux and cross section parameters. Consequently it can significantly reduce systematic uncertainties on the analysis of far detector data. In addition, it measures the cross section of charged current interactions and validates models used by the NEUT generator. There is ongoing work on other cross section measurements, therefore new results are expected in near future. Furthermore, the ND280 has already demonstrated that it can detect $\bar{v}_{\mu}$ interactions and will be able to measure cross sections of anti neutrino interactions.

\section{Acknowledgments}

This work was partially supported by the Polish National Science Center, project number 2011/01/M/ST2/02578.

\section{References}

[1] K. Abe et al., Phys. Rev. Lett. 112, 181801 (2014)

[2] K. Abe et al., Phys. Rev. D. 88, 032002 (2013)

[3] K. Abe et al., Phys. Rev. Lett. 112, 061802 (2014)

[4] K. Abe et al., Nucl. Instr. Meth. A 659, 106 (2011)

[5] The Super-Kamiokande Collaboration, Nucl. Instr. Meth. A 501, 418-462 (2003)

[6] N. Abgrall et al., Nucl. Instr. Meth. A 637, 25-46 (2011)

[7] P-A. Amaudruz et al., Nucl. Instr. Meth. A 696, 1-31 (2012) 
[8] N. Abgrall et al. (NA61/SHINE collaboration), Phys. Rev. C 84, 034604 (2011)

[9] N. Abgrall et al. (NA61/SHINE collaboration), Phys. Rev. C 85, 035210 (2012)

[10] Y. Hayato, Acta Phys. Polon. B 40, 2477 (2009)

[11] K. Abe et al., Phys. Rev. D 89, 092003 (2014)

[12] K. Abe et al., Phys. Rev. D 87, 092003 (2013)

[13] K. Abe et al., arXiv:1407.7389 (2014)

[14] T. Golan et al., Phys. Rev. C 86, 015505 (2012) 